\section{Oxford Textbook of Musculoskeletal Medicine (2nd Edition)}

Michael Hutson and Adam Ward, eds. Oxford: Oxford University Press, 2016, 768 pages, $\$ 325$ US

What is musculoskeletal (MSK) medicine? It certainly is not rheumatology, though rheumatologists with an open mind to diseases outside the familiar realm of inflammatory arthritides and connective tissue diseases could certainly benefit from increasing their knowledge of this field. Two rheumatologists contributed to this updated multiauthored textbook Oxford Textbook of Musculoskeletal Medicine, first published in 2005. Most authors are British, with many others from Europe and other Commonwealth countries, and a few from the United States. Physiatrists, physiotherapists, osteopaths, sports medicine specialists, and orthopedists are best represented among chapter authors. Many authors' field of expertise is not stated, a significant omission.

Early chapters cover foundational aspects and controversies in the field of manual/MSK medicine. The concept of somatic dysfunction is introduced as a better explanation for syndromes such as nonspecific back pain than structural pathology, which we know correlates poorly with symptoms and disability. Biopsychosocial models of pain are extensively discussed. The wise use of the term "fibromyalgia" is sensitively reviewed. The idea that myofascial pain syndrome is a bogus construct was interesting, and certainly substantiated my impressions regarding the lack of proven efficacy of trigger point injections. I also learned about tensegrity, a term coined by Buckminster Fuller, and its applicability to human biomechanics.

The bulk of the textbook covers the assessment of regional MSK disorders, with excellent illustrations of various diagnostic maneuvers, particularly regarding the shoulder and the sacroiliac joints. The concluding section on management strategies includes interesting segments on patient education, manual medicine, and exercise therapy. Other therapies, including prolotherapy, epidural, and spinal injections will be somewhat foreign to the practice of most mainstream rheumatologists.

I would recommend this textbook to seasoned rheumatologists with an interest in orthopedic medicine and regional MSK syndromes. Physiatrists, physiotherapists, chiropractors, and sports medicine specialists will also find the material intriguing.

PHILIP A. BAER, MDCM, FRCPC, FACR, Chair, Section on Rheumatology, Ontario Medical Association, Toronto, and Editor-in-chief, Journal of the Canadian Rheumatology Association, Newmarket, Ontario, Canada. Address correspondence to Dr. P.A. Baer, 305-1333 Neilson Road, Scarborough, Ontario M1B4Y9, Canada. E-mail: Philip.baer@rogers.com

J Rheumatol 2016;43:6; doi:10.3899/jrheum.160251 